\title{
New Perspectives on the Mismatch Negativity (MMN) Component: An Evolving Tool in Cognitive Neuroscience
}

\author{
Elyse S. Sussman • Valerie L. Shafer
}

Received: 27 May 2014/ Accepted: 30 May 2014/Published online: 15 June 2014

(C) Springer Science+Business Media New York 2014

The mismatch negativity (MMN) component of eventrelated brain potentials has become an important tool for investigating the neurobiological mechanisms underlying auditory perception, memory, attention, speech and music. The interest in MMN as a tool of investigation in Cognitive Neuroscience, and its clinical and scientific applications has been steadily growing during the recent years. The successful use of this non-invasive technique has led to an ever-expanding range of areas of study, including the development of auditory perception and cognition across the lifespan, and impairment of auditory cognition observed in a wide range of disorders, such as specific language impairment, dyslexia, central auditory processing disorders, autism, schizophrenia, aphasia, coma, to name only a few. Increased interest in using MMN as a research tool, and increased focus on its clinical use in detecting and monitoring cerebral and psychological dysfunctions has led to investigations aimed at gaining greater clarification of its neural substrate, and underlying mechanisms, using neuropharmacology and animal models. The papers in this special issue represent a small collection of talks given at the Sixth International Conference on Mismatch Negativity $(\mathrm{MMN})$ and its Clinical and Scientific Application held

This is one of several papers published together in Brain Topographyin the "Special Issue: Mismatch Negativity".

E. S. Sussman $(\bowtie)$

Department of Neuroscience, Albert Einstein College of Medicine, Bronx, NY 10461, USA

e-mail: elyse.sussman@einstein.yu.edu

\section{L. Shafer}

Program in Speech-Language-Hearing Sciences, Graduate Center of the City University of New York, New York, NY, USA
May 1-4, 2012 in New York City, reflecting recent advances and theories of MMN.

History of the MMN meeting In recognition of the MMN as an emerging tool in cognitive and clinical neuroscience, the first International Conference on the Mismatch Negativity and its Scientific and Clinical Applications was organized in Helsinki, Finland in 1998 hosted by Risto Näätänen. The first MMN conference was reviewed in a special issue of the journal Audiology and Neuro-otology (Vol. 5, 2000). The success of this event motivated the initiation of subsequent meetings, held every third year, to provide a forum for the exchange of ideas between basic and clinical scientists, postdoctoral fellows, and students, and to increase visibility of the importance of this electrophysiological tool in scientific investigations of auditory processes and their disorders. Thus, following the initial meeting in Helsinki, Finland, the second MMN meeting was held in Barcelona, Spain in 2000; the third in Lyon, France in 2003; the fourth in Cambridge, England in 2006 (reviewed in Psychophysiology, Vol. 21, 2007); the fifth in Budapest, Hungary in 2009; the sixth in New York City, USA in 2012 (papers reflected in this issue); and the seventh MMN conference is expected to be held in Leipzig, Germany in 2015.

The sixth conference (New York, USA) brought together researchers and students from 20 countries around the globe (Fig. 1), who share interests in using MMN as a tool to further the understanding of perceptual and cognitive mechanisms indexed by MMN, disorders of auditory cognition, neurobiological modeling of MMN, and methodological and analysis strategies for MMN application. The papers in this Special Issue represent a sample of this wide range of topics, highlighting recent advances in Methodological issues (C. Hermann, M-H. Giard); neural mechanisms of MMN (B. Gaese, Y. Fishman, M. Malmierca; 


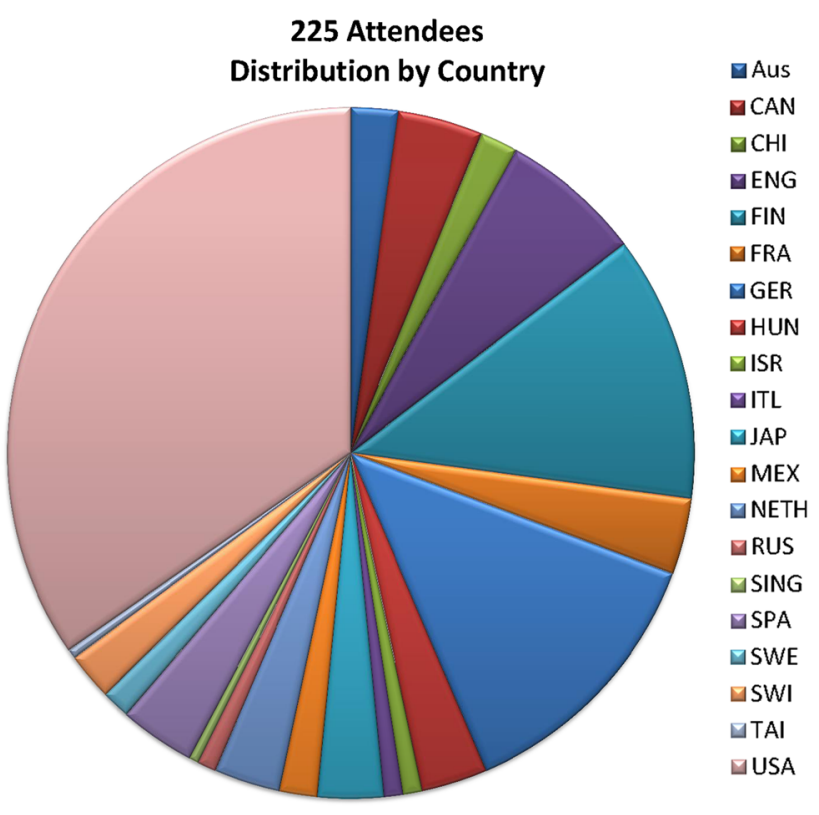

Fig. 1 Distribution of attendees by country. 225 faculty, postdoctoral fellows, and students were in attendance at the Sixth International Conference on Mismatch Negativity (MMN) and its Clinical and Scientific Application from 20 countries around the globe

Clinical issues (D. Morlet, R. Näätänen); and various models of MMN (C. Chandrasekaran, I. Czigler, C. Escera, E. Schröger, E. Sussman, J. Todd).

The meeting began with a series of workshops on basic MMN methods and analysis strategies. Two of the talks from these workshops are reflected in this special issue. Giard et al. (Scalp current density mapping in the analysis of Mismatch Negativity paradigms) describe the advantages of using Scalp Current Density Analysis (SCD) in MMN analysis, and provide a conceptual and mathematical description of SCDs. Herrmann et al. (Time-frequency analysis of event-related potentials: A brief tutorial) provide a tutorial on wavelet analysis, detailing the benefits of this complementary time-frequency analysis for interpreting the event-related potentials.

For clinical applications, Näätänen et al. (Mismatch negativity (MMN) as an index of cognitive dysfunction) provided a review detailing how MMN has been used to index auditory processing capability in a range of neuropsychiatric, neurological and neurodevelopmental disorders. They additionally provide a brief but comprehensive history of the MMN, and suggest future directions for research that could lead to improvements in the use of MMN as a clinical tool. Of particular recent interest is the use of the MMN in prediction of psychosis onset and of return of consciousness in coma and persistent-vegetativestate (PVS) patients. Morlet et al. (MMN and Novelty P3 in Coma and Other Altered States of Consciousness: A Review) specifically focused on the search for markers of consciousness, using the unresponsive patients (chronic vegetative or minimally conscious states) as a model.

With increased interest in using MMN paradigms to investigate clinical disorders, there has been a growing interest in gaining a greater understanding of the neural mechanisms of MMN. This has led to investigations of deviance detection in various animal models, as well as into subcortical contributions to the human scalp-recorded component. One proposed mechanism is stimulus-specific adaptation (SSA), the reduction of the neural response to the frequently repeated stimulus. Malmierca and colleagues and Klein and colleagues assessed SSA in mouse models as a candidate mechanism underlying MMN, and suggested that SSA can account for greater complexity, by investigating more complex stimuli than typically used in animal models (Klein et al. Stimulus-specific adaptation in field potentials and neuronal responses to frequency-modulated tones in the primary auditory cortex)or by investigating SSA expressed in non-lemniscal regions of the medial geniculate body (MGB) of the auditory thalamus(Malmeirca et al. An overview of stimulus-specific adaptation in the auditory thalamus).Fishman (The Mechanisms and Meaning of the Mismatch Negativity) reviewed the extant animal literature on MMN, addressing some controversial issues regarding whether MMN reflects a process of neural adaptation (e.g., SSA) or sensory memory formation. Escera et al. (Deviance detection based on regularity encoding along the auditory hierarchy: electrophysiological evidence in humans) present an emerging view that deviance detection is a basic principle of the functional organization of the auditory system, encoding crucial information at multiple levels of the ascending pathway. Their review provides evidence supporting auditory brainstem and middle latency responses contribution to the MMN deviance detection process. Chandrasekaran et al. (An integrative model of subcortical auditory plasticity) focus their review on the forms of plasticity in the deviance detection process, evidenced in subcortical auditory regions. They propose an integrative model that includes modulation of stimulus input by top-down knowledge, and a mechanism that constantly anticipates and updates incoming stimulus regularities.

The remaining group of review papers explores various models of the MMN system. Sussman et al. (Five myths of $M M N$ : Redefining how to use MMN in clinical and basic research) propose a cortical model of MMN that is based on regularity encoding, dispelling old notions of MMN from long-held beliefs that do not fit with more recent evidence. Schröger et al. (Predictive regularity representations in violation detection and auditory stream segregation: from conceptual to computational models) propose a psychological conceptual framework called the Auditory Event Representation System (AERS), which is founded on 
the assumption that auditory regularity violation detection and the formation of auditory perceptual objects are based on the same predictive regularity representations. Todd et al. (What controls gain in gain control? Mismatch negativity (MMN), priors and system biases) also assess the issue of predictability, investigating how prior context affects deviance detection, focused on understanding how we compute "expectations" for future auditory encounters. Finally, the issue of whether models of auditory deviance detection are analogous in the visual system is still debated. Czigler (Visual mismatch negativity and categorization) reviews the literature on the visual mismatch negativity (vMMN) component, suggesting that the memory system underlying vMMN is sensitive to higher order structures, such as facial characteristics (emotional expression and gender), and not only simple combinations visual features. Thus, suggesting that long-term memory modulates the automatic deviance detection process.

We thank the sponsors of our meeting (Cortech Solutions, Compumedics, Corp., Electrical Geodesic Inc., Brain Products, and Sensorium), and gratefully acknowledge the support received from the National Institutes of Health (R13DC012029) and the National Science Foundation (1156635) for the meeting. We are also thankful for the tireless support of the numerous student volunteers and administrative assistants who helped run and coordinate the meeting, with a special thanks to Miwako Hisagi, Monica Wagner, and Eric Blomquist for helping ensure a successful meeting. We also acknowledge Andrew Hall and Michael Kanan who provided lovely background music, and all the participants for making it such a great experience. Lastly, we thank all of the reviewers of the manuscripts and the authors of the papers for their contribution to this issue, as well as the co-Editors-in-Chief of Brain Topography, Micah Murray and Christoph Michel, who made this issue possible. We hope to see you again in 2015 !

Disclaimer The content of this special issue is solely the responsibility of the authors and does not necessarily represent the official views of the National Institutes of Health or the National Science Foundation.

\section{References}

Chandrasekaran B, Skoe E, Kraus N. An integrative model of subcortical auditory plasticity. Brain Topogr (this issue)

Czigler I. Visual mismatch negativity and categorization. Brain Topogr (this issue)

Escera $\mathrm{C}$ et al. Deviance detection based on regularity encoding along the auditory hierarchy: electrophysiological evidence in humans. Brain Topogr (this issue)

Fishman Y. The Mechanisms and Meaning of the Mismatch Negativity. Brain Topogr (this issue)

Giard M-H et al. Scalp current density mapping in the analysis of Mismatch Negativity paradigms. Brain Topogr (this issue)

Herrmann $\mathrm{C}$ et al. Time-frequency analysis of event-related potentials: A brief tutorial. Brain Topogr (this issue)

Klein $\mathrm{C}$ et al. Stimulus-specific adaptation in field potentials and neuronal responses to frequency- modulated tones in the primary auditory cortex. Brain Topogr (this issue)

Malmierca $\mathrm{M}$ et al. An overview of stimulus-specific adaptation in the auditory thalamus. Brain Topogr (this issue)

Morlet, D. et al. MMN and Novelty P3 in Coma and Other Altered States of Consciousness: A Review

Näätänen $\mathrm{R}$ et al. Mismatch negativity (MMN) as an index of cognitive dysfunction. Brain Topogr (this issue)

Schröger E et al. Predictive regularity representations in violation detection and auditory stream segregation: from conceptual to computational models. Brain Topogr (this issue)

Sussman E et al. Five myths of MMN: Redefining how to use MMN in clinical and basic research. Brain Topogr (this issue)

Todd $\mathrm{J}$ et al. What controls gain in gain control? Mismatch negativity (MMN), priors and system biases. Brain Topogr (this issue) 